3. Department of Health. The expert patient: a new approach to chronic disease management for the 2Ist century. London (UK): The Department; 200I.

4. Clinical practice guidelines and conflict of interest. CMAJ 2005;I73(II):I297.

5. Crawford MJ, Rutter D, Manley C, et al. Systematic review of involving patients in the planning and development of health care. BMJ 2002;325(7375): I263.

DOI:I0.1503/cmaj.106024I

\section{Sudden infant death}

\section{syndrome}

The review article about sudden infant death syndrome (SIDS), by Carl Hunt and Fern Hauck, ${ }^{1}$ was enlightening. However, it was somewhat discouraging in its equivocal negation of the utility of postdelivery electrocardiography. It is the contention of this observer that for any baby with a family history of sudden cardiac death, with evidence of conduction defects or ventricular arrhythmia on cardiorespiratory monitoring, or exhibiting apnea neonatorum, investigations must include electrocardiography to test for cardiac channelopathy such as short QT syndrome, long QT syndrome, Brugada syndrome or progressive cardiac conduction defect, any of which would exclude the diagnosis of SIDS.

\section{John Morphet \\ Cardiologist \\ Niagara-on-the-Lake, Ont.}

\section{REFERENCE}

I. Hunt CE, Houck FR. Sudden infant death syndrome. $C M A J$ 2006;174:I86I-9.

DOI:I0.1503/cmaj.ro6or4I

\section{[The authors respond:]}

We appreciate John Morphet's letter and are pleased to respond. In our article, we stated that electrocardiography is not recommended as a routine strategy for assessing future risk of sudden infant death syndrome. ${ }^{1}$ The cost-effectiveness of such screening in infants without a family history of sudden cardiac death has been studied, but the results have been mixed. The authors of the only North American study concluded that electrocardiography was indicated only in selected groups of in- fants, such as those with symptoms related to apnea or bradycardia or with a family history of sudden infant death syndrome or long QT syndrome. ${ }^{2}$

Both short QT syndrome and long QT syndrome have been reported to cause sudden unexpected death in infancy. ${ }^{2,3}$ Our review did not address the indications for electrocardiography in infants with a family history of sudden cardiac death, but we agree that any baby with a family history of sudden unexpected death in infancy, sudden cardiac death, conduction defect or ventricular arrhythmia should undergo electrocardiography.

It was also outside the scope of our review to discuss recommendations for the clinical evaluation of infants who have experienced an apparent lifethreatening event. In a recent review of the yield of diagnostic testing in 243 such infants, electrocardiography was considered to be indicated in only $25 \%$ 